\title{
Sleep stability and transitions in patients with idiopathic REM sleep behavior disorder and patients with Parkinson's disease
}

Christensen, Julie Anja Engelhard; Jennum, Poul; Koch, Henriette; Frandsen, Rune; Zoetmulder, Marielle; Arvastson, Lars Johan; Christensen, Søren Rahn; Sørensen, Helge Bjarup Dissing

Published in:

Clinical Neurophysiology

Link to article, DOI:

10.1016/j.clinph.2015.03.006

Publication date:

2016

Document Version

Peer reviewed version

Link back to DTU Orbit

Citation $(A P A)$ :

Christensen, J. A. E., Jennum, P., Koch, H., Frandsen, R., Zoetmulder, M., Arvastson, L. J., Christensen, S. R., \& Sørensen, H. B. D. (2016). Sleep stability and transitions in patients with idiopathic REM sleep behavior disorder and patients with Parkinson's disease. Clinical Neurophysiology, 127(1), 537-543.

https://doi.org/10.1016/j.clinph.2015.03.006

\section{General rights}

Copyright and moral rights for the publications made accessible in the public portal are retained by the authors and/or other copyright owners and it is a condition of accessing publications that users recognise and abide by the legal requirements associated with these rights.

- Users may download and print one copy of any publication from the public portal for the purpose of private study or research.

- You may not further distribute the material or use it for any profit-making activity or commercial gain

- You may freely distribute the URL identifying the publication in the public portal 


\title{
Sleep stability and transitions in patients with idiopathic REM sleep behavior disorder and patients with Parkinson's disease ${ }^{\text {th }}$
}

\author{
Julie Anja Engelhard Christensen a,b,c,*, Poul Jennum ${ }^{\mathrm{b}}$, Henriette Koch ${ }^{\mathrm{a}}$, Rune Frandsen ${ }^{\mathrm{b}}$, \\ Marielle Zoetmulder ${ }^{\mathrm{b}, \mathrm{d}}$, Lars Arvastson ${ }^{\mathrm{c}}$, Søren Rahn Christensen ${ }^{\mathrm{c}}$, Helge Bjarrup Dissing Sorensen ${ }^{\mathrm{a}}$ \\ ${ }^{a}$ Department of Electrical Engineering, Technical University of Denmark, Kongens Lyngby, Denmark \\ ${ }^{\mathrm{b}}$ Danish Center for Sleep Medicine, Department of Clinical Neurophysiology, University of Copenhagen, Glostrup Hospital, Glostrup, Denmark \\ ${ }^{\mathrm{c}} \mathrm{H}$. Lundbeck A/S, Copenhagen, Denmark \\ ${ }^{\mathrm{d}}$ Department of Neurology, Bispebjerg Hospital, Copenhagen, Denmark
}

\section{A R T I C L E I N F O}

\section{Article history:}

Accepted 10 March 2015

Available online $\mathrm{xxxx}$

\section{Keywords:}

Parkinson's disease

Rapid eye movement sleep behavior

disorder

Polysomnography

Sleep transitions

Data-driven detection

\section{H I G H L I G H T S}

- Wake and sleep stability is affected in early stages of Parkinson's disease.

- An automated REM stability index is a potential biomarker for Parkinson's disease.

- We discuss problems linked to micro-sleep and nocturnal sleep fragmentation.

\begin{abstract}
A B S T R A C T
Objective: Patients with idiopathic rapid eye movement (REM) sleep behavior disorder (iRBD) are at high risk of developing Parkinson's disease (PD). As wake/sleep-regulation is thought to involve neurons located in the brainstem and hypothalamic areas, we hypothesize that the neurodegeneration in $\mathrm{RBD} /$ PD is likely to affect wake/sleep and REM/non-REM (NREM) sleep transitions.

Methods: We determined the frequency of wake/sleep and REM/NREM sleep transitions and the stability of wake (W), REM and NREM sleep as measured by polysomnography (PSG) in 27 patients with PD, 23 patients with iRBD, 25 patients with periodic leg movement disorder (PLMD) and 23 controls. Measures were computed based on manual scorings and data-driven labeled sleep staging.

Results: Patients with PD showed significantly lower REM stability than controls and patients with PLMD. Patients with iRBD had significantly lower REM stability compared with controls. Patients with PD and RBD showed significantly lower NREM stability and significantly more REM/NREM transitions than controls. Conclusions: We conclude that W, NREM and REM stability and transitions are progressively affected in iRBD and PD, probably reflecting the successive involvement of brain stem areas from early on in the disease. Significance: Sleep stability and transitions determined by a data-driven approach could support the evaluation of iRBD and PD patients.
\end{abstract}

(c) 2015 International Federation of Clinical Neurophysiology.. Published by Elsevier Ireland Ltd. All rights reserved.
Abbreviations: AASM, American Academy of Sleep Medicine; DCSM, Danish Center for Sleep Medicine; EOG, electrooculography; iRBD, Idiopathic rapid eye movement sleep behavior disorder; MSLT, Multiple sleep latency test; NREM, Nonrapid eye movement sleep; PD, Parkinson's disease; PLMD, Periodic leg movement disorder; PSG, Polysomnography; RBD, Rapid eye movement sleep behavior disorder; REM, Rapid eye movement sleep; RSWA, REM sleep without atonia; SLD, Sublaterodorsal tegmental nucleus; SS, Sleep spindles; W, Wake.

Institution at which the work was performed: Danish Center for Sleep Medicine, Department of Clinical Neurophysiology, Glostrup Hospital, Glostrup, Denmark.

* Corresponding author at: Technical University of Denmark (work), Orsteds Plads, Building 349, 2800 Kongens Lyngby, Denmark. Tel.: +45 45255737 (work), Mobile: +1 6507718073 (private).

E-mail address: julie.a.e.christensen@gmail.com (J.A.E. Christensen).

\section{Introduction}

Parkinson's disease (PD) and other synucleinopathies are debilitating diseases with impacts on morbidity, mortality, work, and social and family life. The disorders have large direct and indirect costs for society (Jennum et al., 2011). PD is the second most common neurodegenerative disease after Alzheimer's disease. Treatment is purely symptomatic and does not alter underlying disease progression (Schapira et al., 2013). When motor symptoms are present, alterations of the substantia nigra with reduced production or depletion of dopamine is found (Galvin et al., 2001), 
mediating the classical PD phenotype. Over the last 20 years, it has become increasingly clear that the entire brain is affected by the pathology, which typically starts in caudal areas of the brainstem and progresses anteriorly (Braak et al., 2003).

Recent research has focused on early detection of PD, notably changes in sleep-wake pattern and rapid eye movement (REM) sleep without atonia (RSWA) coinciding with dream-enacting behavior - REM sleep behavior disorder (RBD) (Salawu et al., 2010). RBD is closely associated with PD (Munhoz and Teive, 2014), and patients suffering from idiopathic RBD (iRBD) are at great risk of subsequently developing Parkinsonism or dementia (Schenck et al., 2013, 2003, 1996). Several studies have focused on analysis of sleep data in the search for PD biomarkers (Dos Santos et al., 2014). These have examined measures of sleep spindle densities, RSWA, slow wave characteristics (Christensen et al., 2014b; Kempfner et al., 2014a,b; Latreille et al., 2014, 2011; Postuma et al., 2010) and other measures of abnormalities of brain stem function, including autonomic functions such as heart-rate variability (Sorensen et al., 2013a, 2012) and other non-motor symptoms (Garcia-Ruiz et al., 2014; Sakakibara et al., 2014).

Sleep is strongly regulated by groups of neurons located in the brainstem and midbrain areas, which form reciprocal connections (Luppi et al., 2011; Saper et al., 2010, 2001; Schwartz and Roth, 2008). These "sleep-wake switches" are mutually dependent and have been referred to as the wake-sleep and REM-NREM sleep switches, respectively. Despite the mutually inhibitory loops involved in the two switching mechanisms, if either side of the two loops is weakened or injured, unwanted instability can occur in either of the states, irrespective of which side is damaged (Schwartz and Roth, 2008). As neurons of the brain stem and basal brain structures are affected in synucleinopathies (Braak et al., 2003), we propose that the neurodegeneration will have a progressive impact on the wake-sleep and REM-NREM transitions and stability. Manual scoring of sleep in patients with PD is not very reliable and prone to high inter- and intra-rater variability (Danker-Hopfe et al., 2004; Jensen et al., 2010). Therefore, this study analyzed wake-sleep and REM-NREM transitions as well as W, REM and NREM stability measures based on automatically identified as well as manually scored REM, NREM and W stages. The automatic method used has been validated by (Koch et al., 2014) using the same PSG data as analyzed in this study.

\section{Methods}

\subsection{Subjects and recordings}

Subjects were recruited from the Danish Center for Sleep Medicine (DCSM) in the Department of Clinical Neurophysiology, Glostrup University Hospital in Denmark. A total of 27 patients with PD, 23 patients with iRBD, 25 patients with periodic leg movement disorder (PLMD) and 23 control subjects aged 40 years or more and with no history of movement disorder, dream-enacting behavior or other previously diagnosed sleep disorders were included. Nineteen of the patients with PD had $\mathrm{RBD}\left(\mathrm{PD}^{+}\right)$and eight did not $\left(\mathrm{PD}^{-}\right)$, as determined by the presence of RSWA as well as clinical complaints. All or a subset of the subjects included here have appeared in previous studies (Christensen et al., 2014a,b; Sorensen et al., 2013a; Zoetmulder et al., 2014a,b). Patient evaluations included a comprehensive medical and medication history and a polysomnography (PSG) analyzed according to the American Academy of Sleep Medicine (AASM) standard (Iber et al., 2007). A multiple sleep latency test (MSLT) was performed in any cases where narcolepsy was suspected. The PD diagnose rely on clinical features including motor information typically for PD which further includes DAT scan and in some cases also MRI of the brain. The RBD Screening Questionnaire (RBDSQ) (StiasnyKolster et al., 2007) was used to screen for RBD, and the iRBD patients were divided in two groups: (1) those with a total score of nine or less (iRBD ${ }^{-}$) and (2) those with a total score of 10 or more $\left(\mathrm{iRBD}^{+}\right)$. The cutoff was chosen to divide the iRBD patients into those with major self-reported dream enactments and those with minor. All iRBD patients thus had self-reported dream enactment as well as RSWA in the recorded night analyzed. Patients treated with medication known to affect sleep stages (antidepressants, antipsychotics, hypnotics) were excluded, except for dopaminergic treatments. We are fully aware of the potential effect of dopaminergic drugs on vigilance (Micallef et al., 2009), but discontinuation of dopaminergic treatment in actively treated patients prior to PSG also risks deleterious discontinuation effects. Furthermore, discontinuation is very difficult to achieve in clinical settings and may have unpleasant and negative motor effects that could interfere with the study and might even be unethical. One patient in the PD group later developed Multiple System Atrophy and another patient with PD developed Lewy Body Dementia. Controls underwent at least one night of PSG as an outpatient. Patients underwent at least one night of PSG as an outpatient or in hospital in accordance with the AASM standard (Iber et al., 2007). The quality of each PSG data set was individually evaluated, and recordings were discounted if channels became disconnected or were significantly contaminated with artifacts. Low-quality PSGs were repeated whenever possible. Demographic data as well as PSG variables for the six groups are summarized in Table 1.

\subsection{Automatic staging of sleep}

Sleep changes in both microarchitecture and macroarchitecture have been reported in neurodegenerative disease, the former interfering with sleep scoring and increasing intra- and inter-rater variability. Specifically, patients with iRBD and PD have been reported to have EEG frequency slowing (Rodrigues Brazète et al., 2013), changed EEG during REM sleep (Christensen et al., 2014a; Fantini et al., 2003; Hansen et al., 2013), changed morphology or fewer rapid or slow eye movements (Christensen et al., 2014a, 2013) and sleep spindles (SS) (Christensen et al., 2014b; Latreille et al., 2014), and display RSWA. The listed findings contribute to very altered sleep and consequently, sleep stage scoring this pathology is associated with high inter-rater variability (Danker-Hopfe et al., 2004; Jensen et al., 2010). To overcome this, an automatic sleep detector was used to identify REM, NREM and W for each subject and analyses were performed using automatically scored sleep stage data. The automatic sleep scoring technique used in this study has been validated on the same PD, iRBD, PLMD and control PSG data set as used in this study, and the methods are described in detail by Koch et al. (2014). Specifically, the method is optimized on nocturnal PSG of 50 subjects, and validated on an additional 76 subjects (a mixture of the same controls and patients included in this study). Our automatic detector is data-driven, identifying sleep states based on the distribution of certain EEG and electrooculographic (EOG) characteristics, which can be interpreted as specific fingerprints for each epoch. Due to the presence of RSWA, the automatic identification approach is based on EEG and EOG characteristics only. Briefly, for each second, EEG C3-A2 and O1-A2 single-sided amplitude spectra in clinical frequency bands as well as EOG power below $5 \mathrm{~Hz}$ and cross-correlations between the two EOG channels are computed. Surveying these measures simultaneously in three-second intervals with a step size of one second, the approach identifies patterns indicative of the various sleep stages. The method produces a mixture of probabilities for the different sleep stages, and final identification of NREM, REM and $\mathrm{W}$ is based on the highest probability when combining probabilities of individual sleep stages (Koch et al., 2014). A clear 
Table 1

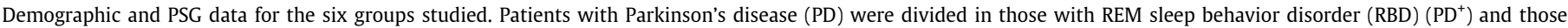

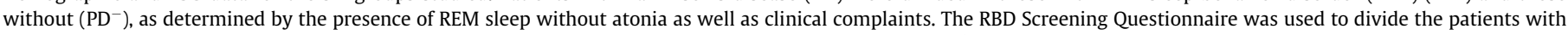

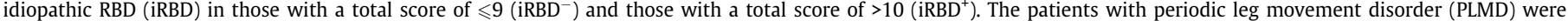
included as a secondary control group. The disease onset is stated as years from clinical diagnosis (PD patients) or self-reported subjective RBD-symptoms (iRBD patients).

\begin{tabular}{|c|c|c|c|c|c|c|}
\hline & Controls & PLMD & $\mathrm{iRBD}^{-}$ & $\mathrm{iRBD}^{+}$ & $\mathrm{PD}^{-}$ & $\mathrm{PD}^{+}$ \\
\hline Total counts (Male/Female) & $23(7 / 16)$ & $25(13 / 12)$ & $12(9 / 3)$ & $11(10 / 1)$ & $8(5 / 3)$ & $19(13 / 6)$ \\
\hline $\begin{array}{l}\text { Age } \\
\qquad \text { [years, } \mu \pm \sigma]\end{array}$ & $56.7 \pm 9.2$ & $56.9 \pm 11.6$ & $61.8 \pm 6.8$ & $66.3 \pm 7.2$ & $68.8 \pm 8.4$ & $63.7 \pm 6.7$ \\
\hline $\begin{array}{l}\text { BMI } \\
\qquad\left[\mathrm{kg} / \mathrm{m}^{2}, \mu \pm \sigma\right]\end{array}$ & $23.1 \pm 2.5$ & $25.7 \pm 3.8$ & $25.1 \pm 2.6$ & $25.9 \pm 3.6$ & $24.1 \pm 3.6$ & $25.9 \pm 2.9$ \\
\hline $\begin{array}{l}\text { Sleep Efficiency } \\
\qquad[\%, \mu \pm \sigma]\end{array}$ & $86.2 \pm 10.9$ & $86.1 \pm 7.5$ & $82.5 \pm 7.4$ & $85.8 \pm 6.7$ & $67.9 \pm 15.5$ & $80.3 \pm 9.9$ \\
\hline $\begin{array}{l}\text { Time in Bed } \\
\qquad[\mathrm{min}, \mu \pm \sigma]\end{array}$ & $484 \pm 81.2$ & $431 \pm 50.0$ & $518 \pm 107.3$ & $474 \pm 71.1$ & $427 \pm 53.8$ & $461 \pm 88.3$ \\
\hline W $[\%, \mu \pm \sigma]$ & $13.8 \pm 10.9$ & $13.9 \pm 7.5$ & $17.5 \pm 7.4$ & $14.2 \pm 6.7$ & $32.1 \pm 15.5$ & $19.7 \pm 9.9$ \\
\hline $\begin{array}{l}\text { REM } \\
\qquad[\%, \mu \pm \sigma]\end{array}$ & $18.9 \pm 6.6$ & $18.5 \pm 6.1$ & $17.7 \pm 7.1$ & $16.3 \pm 7.1$ & $9.3 \pm 5.3$ & $14.6 \pm 10.8$ \\
\hline $\begin{array}{l}\mathrm{N} 1 \\
{[[\%, \mu \pm \sigma]}\end{array}$ & $8.8 \pm 4.6$ & $8.4 \pm 7.2$ & $7.5 \pm 2.6$ & $10.8 \pm 5.4$ & $7.3 \pm 6.0$ & $12.6 \pm 9.9$ \\
\hline${ }^{\mathrm{N} 2}[\%, \mu \pm \sigma]$ & $44.7 \pm 9.4$ & $44.7 \pm 10.9$ & $40.0 \pm 10.9$ & $43.0 \pm 10.3$ & $39.2 \pm 12.9$ & $39.3 \pm 15.1$ \\
\hline $\begin{array}{l}\mathrm{N} 3 \\
{[[\%, \mu \pm \sigma]}\end{array}$ & $13.8 \pm 7.3$ & $14.5 \pm 9.1$ & $17.3 \pm 9.9$ & $15.7 \pm 11.2$ & $12.1 \pm 9.1$ & $13.9 \pm 17.6$ \\
\hline $\begin{array}{l}\text { LM index } \\
\quad[\text { no/hour, } \mu \pm \sigma]\end{array}$ & $18.1 \pm 13.1$ & $56.5 \pm 36.8$ & $49.9 \pm 32.0$ & $31.6 \pm 22.9$ & $39.2 \pm 40.1$ & $42.7 \pm 44.4$ \\
\hline $\begin{array}{l}\text { PLM index } \\
\qquad[\text { no/hour, } \mu \pm \sigma]\end{array}$ & $7.7 \pm 7.4$ & $36.5 \pm 24.6$ & $14.9 \pm 16.3$ & $23.0 \pm 17.1$ & $11.2 \pm 11.5$ & $10.8 \pm 10.3$ \\
\hline $\begin{array}{l}\text { Disease duration } \\
\text { [years, } \mu \pm \sigma \text { ] }\end{array}$ & NA & NA & $5.3 \pm 9.8$ & $11.3 \pm 12.0$ & $7.7 \pm 5.7$ & $4.6 \pm 3.3$ \\
\hline $\begin{array}{l}\text { RBD score } \\
\qquad[\mu \pm \sigma]\end{array}$ & NA & NA & $7.7 \pm 1.6$ & $11.1 \pm 1.0$ & $2.5 \pm 1.1$ & $9.7 \pm 2.3$ \\
\hline
\end{tabular}

advantage of this approach is that it takes distributions of microsleep characteristics into account, and each sleep epoch is labeled individually and independently of adjacent epochs. In spite of the pace of the sleep-regulating loops, the sleep transitions happen gradually, and the method used will assign an epoch a stage once the probability of this stage becomes the dominant one, and not when only few distinct signs of a stage is shown. It is also one of the reasons why the manually and automatically scored stages differ. The overall mean accuracy rates for detecting NREM, REM and W ranged from $70 \%$ for patients with PD to $77 \%$ for control subjects when comparing with manually single-scored hypnograms.

\subsection{Analysis of transitions and stability}

Two transition measures and three stability measures were defined and analyzed in this study. Wake-sleep transitions were defined as the number of shifts from any sleep stage (N1, N2, N3, REM) to wakefulness or vice versa. REM-NREM transitions were defined as the number of shifts from REM sleep to any NREM sleep stage $(\mathrm{N} 1, \mathrm{~N} 2, \mathrm{~N} 3)$ or vice versa. Both measures were defined as the frequency per minute of total time in bed. The three stability measures were defined as the number of passages/toggles 1) from a REM stage to a REM stage (REM $\rightarrow$ REM), 2) from any NREM stage to any NREM stage (NREM $\rightarrow$ NREM) or 3 ) from a W stage to a W stage $(\mathrm{W} \rightarrow \mathrm{W})$. These measures were defined as the frequency of passages between two REM, NREM or $W$ epochs per minute of the total time spent in these stages, respectfully. For each subject, transition and stability measures were computed based on the manually scored hypnogram as well as the automatic REM/ NREM/W staging technique. Wilcoxon rank-sum tests were performed to compare the between-group transition and stability measures, yielding 15 comparisons for each measure summing up to 75 tests in total. A significance level of $p<0.05$ was used. The Benjamini-Hochberg procedure was used to correct for multiple testing using a false discovery rate at level $q=0.10$.

\section{Results}

Transition and stability measures computed from the automatic identified NREM, REM and $\mathrm{W}$ are illustrated in Fig. 1, and the results found from the manually scored hypnogram are illustrated in Fig. 2. Mean and standard deviations are illustrated in bar plots to the left and measures for each subject illustrated as dots to the right.

The results obtained from the automatically identified stages showed that patients with iRBD with a RBDSQ total score of nine or lower had significantly lower REM stability compared with controls $(p=0.0106)$. Patients with iRBD with a RBDSQ total score of ten or more had significantly lower REM stability compared with controls $(p=0.0036)$ and PLMD patients $(p=0.0091)$. PD patients without RBD showed significantly lower REM stability compared to the controls $(p<0.0055)$ and PLMD $(p<0.0052)$. PD patients with RBD had significantly lower REM stability than controls $(p=0.0003)$ and PLMD patients $(p=0.0004)$, significantly lower NREM stability than controls $(p=0.0050)$, and significantly more REM-NREM sleep transitions than controls $(p=0.0107)$. Finally, iRBD patients with a RBDSQ total score of nine or lower showed a trend towards lower REM stability compared with PLMD patients, and iRBD patients with a RBDSQ total score of ten or more showed a trend of more REM-NREM sleep transitions than controls. However, these trends were not statistically significant after correction for multiple testing. No significant differences were found between any of the iRBD and PD groups, although PD patients showed a trend towards lower REM stability and more REM-NREM sleep transitions compared with iRBD patients.

No significant between-group differences were found for the transition and stability measures computed from the manually scored hypnograms. It is seen that the stability measures computed from the manually scored hypnogram are all greater than the measures computed from the data-driven sleep staging technique. The measures for REM-NREM sleep transitions are greater for the automatic staging technique compared to the manually 

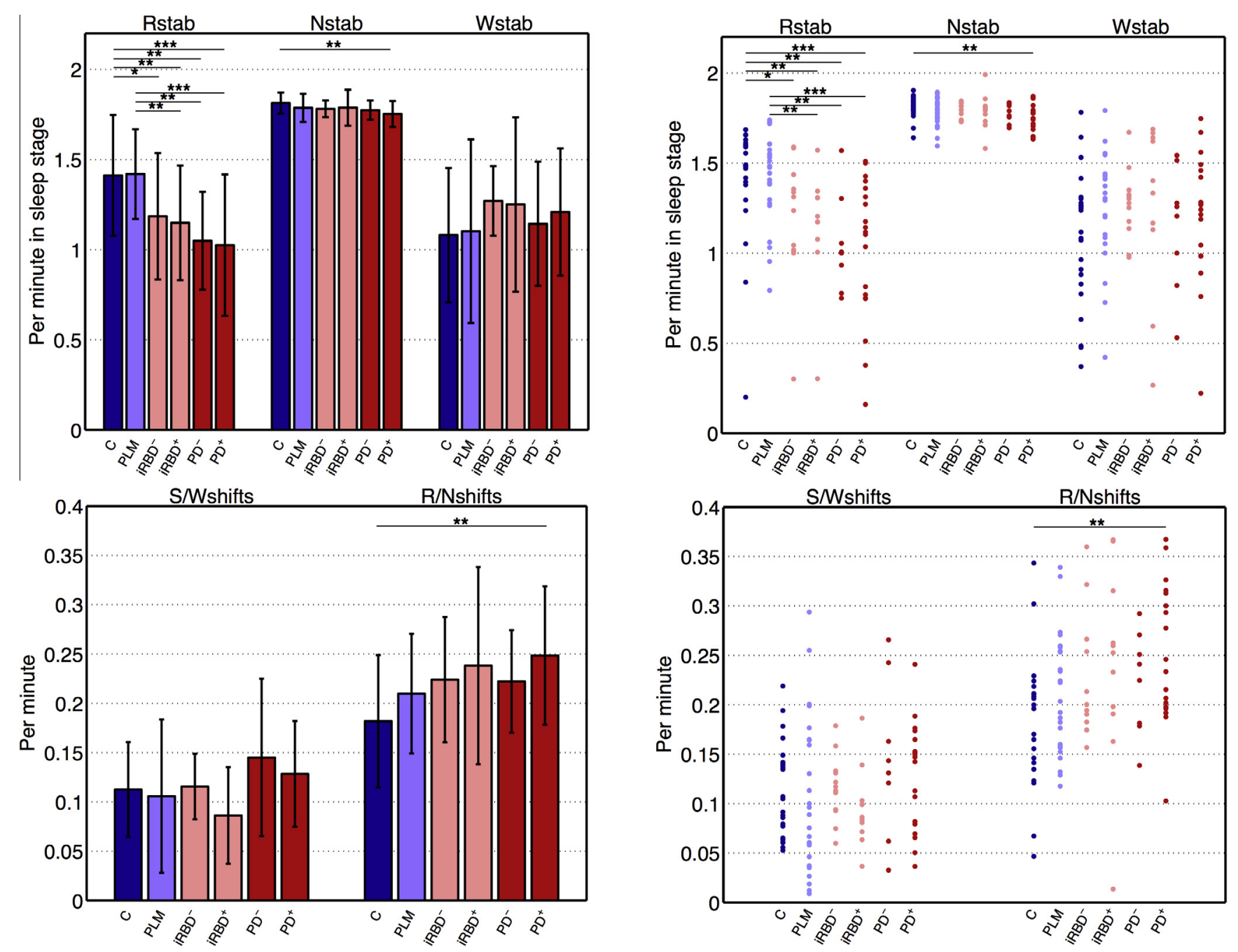

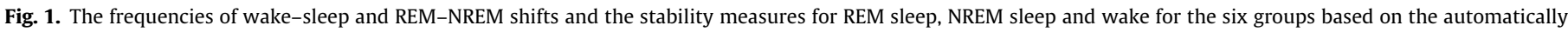

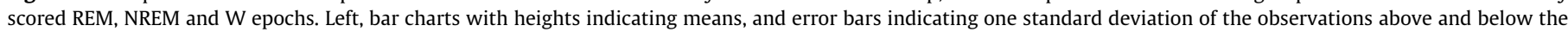

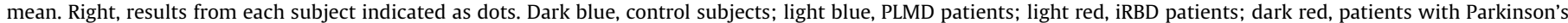

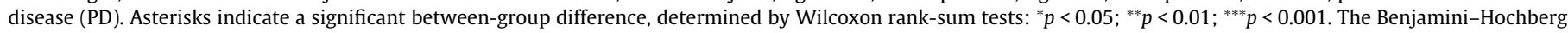

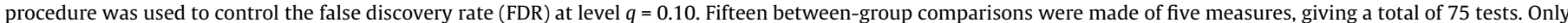

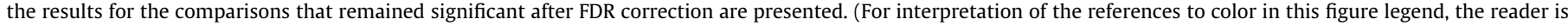
referred to the web version of this article.)

scored sleep stages, and the differences between the REM stability measures are thought to be the main cause hereof.

\section{Discussion}

This study is the first to analyze wake-sleep and REM-NREM transitions and the stability of REM, NREM and $W$ in PD, iRBD and PLMD patients compared with controls. Furthermore, we divided the PD patients into those with and those without RBD, and the iRBD patients were divided in those with a high total score on the RBDSQ and those with a low score. Our main findings are: (1) REM sleep is less stable in iRBD and PD patients than in PLMD patients and control subjects, regardless of the RBDSQ total score and the presence of RBD. A non-significant trend was seen for a lower REM sleep stability in PD compared to iRBD patients. (2) PD patients with RBD showed significantly lower NREM stability and significantly more REM-NREM shifts than controls. Same trend, however non-significant, was seen for iRBD with high scores in the RBDSQ compared with control subjects. Overall, trends were seen for lower REM stability and more REM/NREM transitions in both groups of iRBD and PD patients. These results indicate that the ability to maintain REM and NREM sleep is a biomarker for iRBD and PD, regardless of the presence of clinical symptoms.

The sub-division of iRBD and PD patients might not be optimal, as it does not reflect the actual RBD severity in the recording analyzed. The PD patients without RBD might have RSWA to a certain degree but no RBD diagnosis as they lack to report subjective symptoms. Contradictory, the iRBD patients with major selfreported dream enactments might show just sufficient RSWA to be diagnosed. Analyzing trends for the stability and transitional measures as a function of clinical RBD severity could give a better insight in how correlated the features are with RBD severity. The clinical RBD severity scale reported in (Sixel-Döring et al., 2011) analyzes RBD on an event-to-event basis, including both vocalization and movements, and could be used in future studies to look for such trends and correlations.

Wake-sleep and REM-NREM shifts as well as stability measures were computed based on the manually scored hypnogram as well as a data-driven identification of REM, NREM and W stages. A datadriven model recognizes the underlying structure of the data and automatically identifies wake and sleep stages. Using a data-driven sleep staging approach has several advantages over manual scoring. 

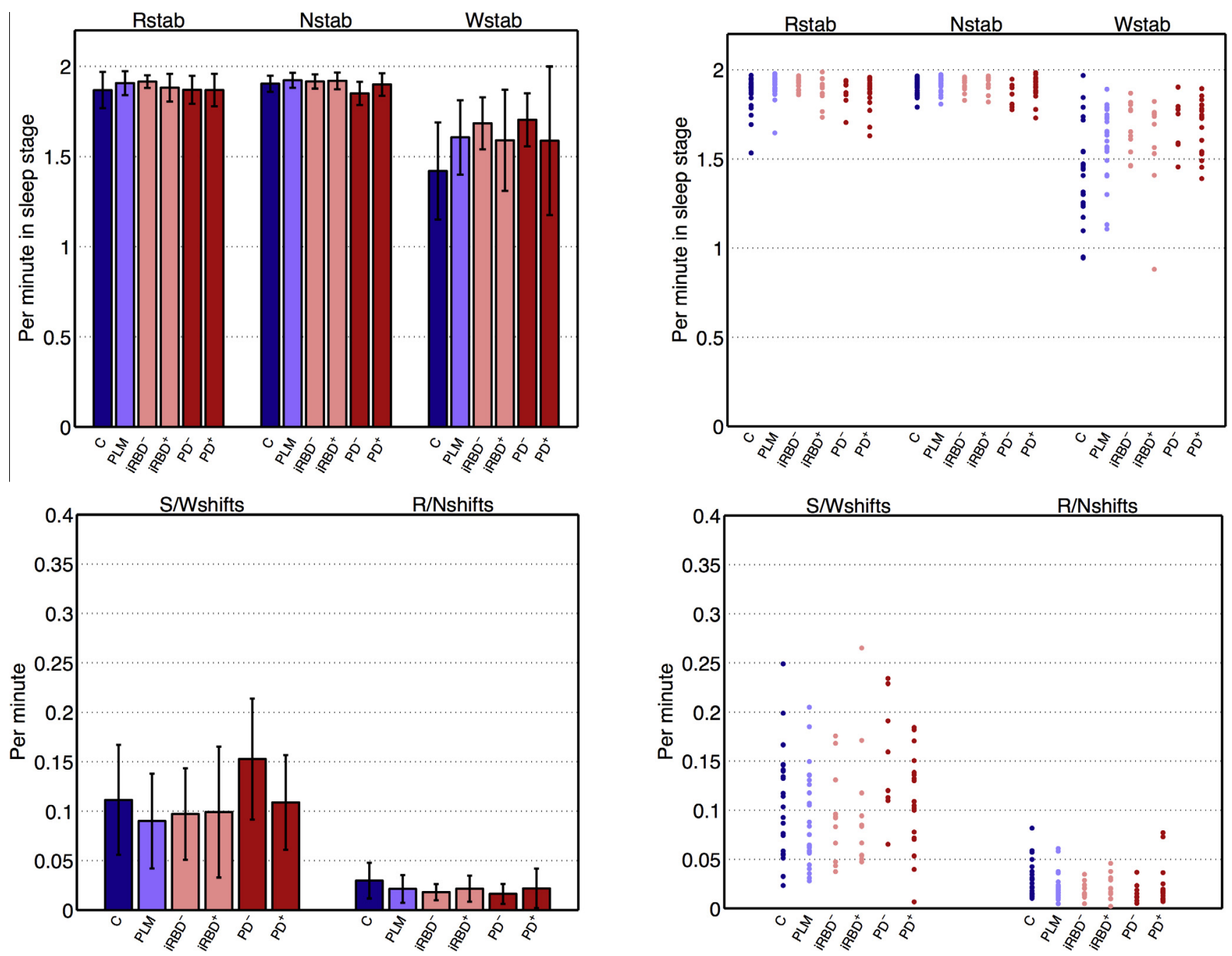

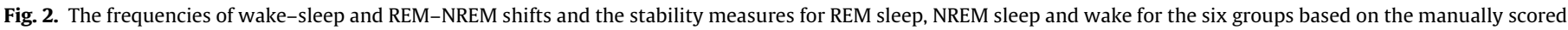

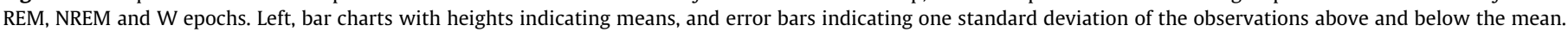

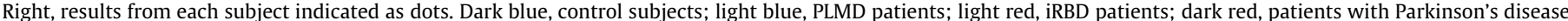

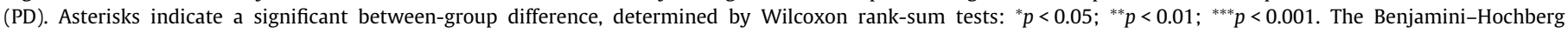

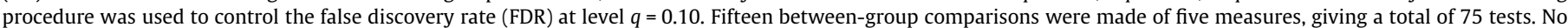

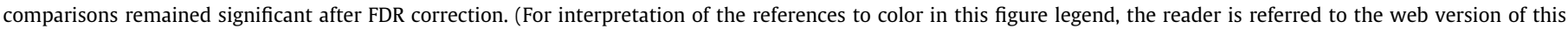
article.)

First, automatic staging encompasses micro-sleep characteristics delineated over time intervals as short as one second; the dynamics over such short intervals are not captured by manual scoring, which is of a much lower resolution. The model looks deeper into each sleep epoch and can survey and identify many more sleep characteristics than the human eye can assess. Although final identification is made for 30-s intervals in the automatic detection as well, decisions are based on changes or transitions occurring at a much faster rate. Second, using an automatic, data-driven approach, sleep stages across the night as well as across subjects are more comparable as they are obtained using exactly the same algorithm. Third, using the automatic model, identification of each epoch is based solely on micro-sleep characteristics and changes within the epoch in question, rather than on the scoring of adjacent epochs. As the model features the analysis of short time intervals, it enables a highly detailed description of each sleep epoch, which reduces variation in inter- and intra-subject scoring. Consistent with this, manual scorings for patients with PD have low inter-rater agreement rates (Danker-Hopfe et al., 2004; Jensen et al., 2010).

No significant differences between groups were found for the manually scored hypnograms, whereas the data-driven labeling indicated many between-group differences. The reason for these contradictory results are suggested to be caused by the two different sleep scorings strategies. The fact that the data-driven method makes the labeling solely based on the characteristics of the epoch in question and not the labeling of the prior epoch allows in specific REM sleep to be terminated when no clear EEG and EOG structures supporting REM sleep are present. Contrarily, manually scorings terminate REM sleep only when a clear NREM or $\mathrm{W}$ structure is present (Iber et al., 2007). Specifically, REM sleep is besides the characteristic eye movements identified by atonia and EEG similar to that during wakefulness. In periods where eye movements are not present, but the atonia as well as the aroused EEG is maintained, the manual scoring of REM sleep is continued. Relating this to EMG with lack of atonia and EEG with altered characteristics or lack of clear micro-sleep structures as seen in patients with PD or iRBD (Christensen et al., 2014a,b; Dauvilliers et al., 2007; Fantini et al., 2003; Kempfner et al., 2014a,b; Petit et al., 2004), termination of REM sleep is harder to confirm for a manual scorer. As a result, the manually scorings lack to illustrate differences in the REM stability measure, as well as in the REM-NREM sleep transitions. 
The sleep regulating mechanisms involve several neurons, which are mainly located in the basal brain regions (Luppi et al., 2011; Saper et al., 2001). Our findings suggest that these mechanisms are affected in patients with iRBD and specifically in patients with PD, suggesting iRBD as an intermediate stage between controls and patients with PD, consistent with Braak's staging theory (Braak et al., 2003). Specifically, REM stability was affected, which involves the neurological networks controlling REM-NREM sleep transitions presented as consisting of REM-on and REM-off areas located in the brainstem (Lu et al., 2006; Luppi et al., 2011). The REM-on area is thought to contain two populations of neurons, where one set projects into the basal forebrain and regulates EEG components of REM sleep, and the other projects into the medulla and spinal cord and regulates atonia during REM sleep (Lu et al., 2006). As a diagnostic criterion of RBD is loss of atonia during REM sleep, the neurons regulating atonia must be affected to some degree in these patients. The REM staging and thereby the REM stability measures in this study were only based on EEG and EOG characteristics, suggesting that the neurons regulating the cortical components of REM sleep are also affected, agreeing with previous findings (Fantini et al., 2003). Specifically, it is proposed that the REM-specific EEG structures are signified to a lesser degree in iRBD and PD patients than in controls and PLMD patients. The reason for this could be neurodegeneration of the ascending branch of the SLD neurons themselves or the heavy innervation between the REM-on and REM-off areas. As the descending branch of the SLD neurons are destroyed in iRBD patients (Luppi et al., 2013, 2011), it is very likely that the ascending branch are affected as well, as these neurons are physically adjacent to each other. In addition, the reported changes in dream enactment in these patients support the hypothesis that the medullo-cortical branch is affected as well.

Another reason for the lower stability of REM sleep in iRBD and PD patients could simply be neurodegeneration affecting prethalamic fibers located in the brain stem. This would cause changes in the patterns, density and/or circadian rhythm of thalamicinduced EEG features such as K-complexes, SS and alpha-activity, etc. Impairment of thalamic control would affect not only the overall sleep rhythm, but also the stability of the different sleep stages.

In a former study we showed that sleep transitions are affected in hypocretin-deficient narcolepsy (Sorensen et al., 2013b). This delineates the role of the hypocretin system in wake-sleep regulation and in NREM-REM regulation. Cerebrospinal hypocretin levels are often normal or subnormal but there are fewer hypocretinergic neurons in hypothalamus in PD (Wienecke et al., 2012). Surprisingly, one study found high a level of hypocretin-1 (orexin-A) (Bridoux et al., 2013), but the small number of patients limited further conclusions. There are limited data from iRBD and PD patients concerning the involvement of hypocretin level. However the spinal hypocretinergic level does not need to fully represent the factual loss of hypocretinergic neurons (Compta et al., 2009), and its involvement in RSWA and the role of the SLD are not fully understood, even in hypocretin-deficient narcolepsy (Knudsen et al., 2010).

It has to be noted that the lower REM sleep stability in PD patients can be due to comorbidities, such as e.g. comorbid insomnia; a common symptom in PD. Riemann et al. (Riemann et al., 2012) reports REM sleep instability as an objective measure for primary insomnia patients compared to good sleeper controls. Although they measure REM sleep instability differently than us (number of manually scored micro-arousals and awakenings per hour of REM sleep compared to our fully data-driven method), we cannot rule out insomnia as causative for the lower REM sleep stability. Insomnia is a diagnosis highly based on subjective measurements, and as we focused on electrophysiological measurements alone, we lack a comparison of the presence and degree of comorbid insomnia to the stability and transitional measurements.
Future studies have to address this issue, also including a group of patients with primary insomnia.

A major limitation of this study is that we have not fully investigated the disagreement between the manual and automatic scoring. A weakness of the automatic detector is that it depends on the ability to capture micro-sleep events within the different disease groups. Our "words-in-a-bag" assumption introduces considerable adaptability (each epoch is described by the use of 1192 different "words"), which we believe makes our model capable of better recognizing altered micro-sleep architecture events, such as abnormal SS, K-complexes and eye movements. Optimally, micro-sleep events should be validated and compared in the different groups. However, we do not have a suitable measure of how well the different micro-sleep events are captured. In the case of the control subjects, the manual scores might be a suitable gold standard, although the mean inter-rater agreement rate has been reported to be as low as 76\% (Norman et al., 2000). In the patients' case, the inter-rater agreement rates are lower, and in particularly in patients with $\mathrm{PD}$, where the agreement rate has been reported to be only "fair" in more than $25 \%$ of sleep recordings (DankerHopfe et al., 2004). These results all imply some difficulties with the standard sleep scoring method, whether it be stated as the R\&K or the AASM standard. The standards are based on microand macro-sleep structures in healthy, young subjects, and it forces the sleep rhythm to be explained by five or six discrete stages defined in 30-s windows. Transitions between sleep stages and from sleep to wakefulness might happen faster than is captured by manual scoring. The fixed period of $30 \mathrm{~s}$ is not a physiological parameter, as the brain does not work to a specific or consistent timescale. Analyzing sleep in sleep and/or neurological disorders has proved to be difficult due to disruptions in the sleep transition mechanisms in the brain. Therefore, changes and differences in the micro- and macrostructure of sleep might not be captured by manual scoring with the principles as defined today. The appearance of sleep simply cannot be fitted to the scoring standard.

\section{Conclusions}

In conclusion, our study suggests that patients with iRBD and PD suffer from instability in the wake-sleep and NREM-REM transitions and instability of wake and sleep. These findings are in accordance with the initial hypothesis that iRBD is an early form of PD and that the basal brain is involved in these diseases in early disease stages. Our results further argue for the problems that these patients suffer from micro-sleep and nocturnal sleep fragmentation. Further studies should be conducted to examine these findings in other neurodegenerative diseases affecting wake and sleep regulation.

\section{Acknowledgments}

The PhD project is supported by grants from $\mathrm{H}$. Lundbeck $\mathrm{A} / \mathrm{S}$, the Lundbeck Foundation, the Technical University of Denmark and the Center for Healthy Aging, University of Copenhagen. Conflict of interest: Julie A. E. Christensen, Henriette Koch, Rune Frandsen, Marielle Zoetmulder, Lars Arvastson, Søren R. Christensen, Helge B. D. Sorensen and Poul Jennum: No conflicts of interest reported.

\section{References}

Braak H, Del Tredici K, Rüb U, de Vos RA, Jansen Steur EN, Braak E. Staging of brain pathology related to sporadic Parkinson's disease. Neurobiol Aging 2003;24:197-211.

Bridoux A, Moutereau S, Covali-Noroc A, Margarit L, Palfi S, Nguyen JP, et al. Ventricular orexin-A (hypocretin-1) levels correlate with rapid-eye-movement sleep without atonia in Parkinson's disease. Nat Sci Sleep 2013;12:87-91. 
Christensen JAE, Zoetmulder M, Koch H, Frandsen R, Arvastson L, Christensen SR, et al. Data-driven modeling of sleep EEG and EOG reveals characteristics indicative of pre-Parkinson's and Parkinson's disease. J Neurosci Methods 2014a;235:262-76.

Christensen JAE, Kempfner J, Zoetmulder M, Leonthin HL, Arvastson L, Christensen $\mathrm{SR}$, et al. Decreased sleep spindle density in patients with idiopathic REM sleep behavior disorder and patients with Parkinson's disease. Clin Neurophysiol 2014b;125:512-9.

Christensen JAE, Koch H, Frandsen R, Kempfner J, Arvastson L, Christensen SR, et al. Classification of iRBD and Parkinson's disease patients based on eye movements during sleep. Conf Proc IEEE Eng Med Biol Soc 2013;2013:441-4.

Compta Y, Santamaria J, Ratti L, Tolosa E, Iranzo A, Muñoz E, et al. Cerebrospinal hypocretin, daytime sleepiness and sleep architecture in Parkinson's disease dementia. Brain 2009;132:3308-17.

Danker-Hopfe H, Kunz D, Gruber G, Klösch G, Lorenzo JL, Himanen SL, et al. Interrater reliability between scorers from eight European sleep laboratories in subjects with different sleep disorders. J Sleep Res 2004;13:63-9.

Dauvilliers Y, Rompré S, Gagnon J, Vendette M, Petit D, Montplaisir J. REM sleep characteristics in narcolepsy and REM sleep behavior disorder. Sleep 2007;30:844-9.

Dos Santos AB, Kohlmeier KA, Barreto GE. Are sleep disturbances preclinical markers of Parkinson's disease? Neurochem Res 2014. http://dx.doi.org 10.1007/s11064-014-1488-7. in press.

Fantini ML, Gagnon JF, Petit D, Rompré S, Décary A, Carrier J, et al. Slowing of electroencephalogram in rapid eye movement sleep behavior disorder. Ann Neurol 2003:53:774-80.

Galvin JE, Lee VM, Trojanowski JQ. Synucleinopathies: clinical and pathological implications. Arch Neurol 2001;58:186-90.

Garcia-Ruiz PJ, Chaudhuri KR, Martinez-Martin P. Non-motor symptoms of Parkinson's disease A review. . from the past. J Neurol Sci 2014;338:30-3.

Hansen IH, Marcussen M, Christensen JAE, Jennum P, Sorensen HBD. Detection of a sleep disorder predicting Parkinson's disease. Conf Proc IEEE Eng Med Biol Soc 2013;2013:5793-6.

Iber C, Ancoli-Israel S, Chesson AL, Qua SF. The AASM Manual for the Scoring of Sleep and Associated Events: rules, terminology, and technical specifications. Westchester, IL: American Academy of Sleep Medicine; 2007.

Jennum P, Zoetmulder M, Korbo L, Kjellberg J. The health-related, social, and economic consequences of parkinsonism: a controlled national study. J Neurol 2011;258:1497-506.

Jensen PS, Sorensen HBD, Leonthin HL, Jennum P. Automatic sleep scoring in normals and in individuals with neurodegenerative disorders according to new international sleep scoring criteria. J Clin Neurophysiol 2010;27:296-302.

Kempfner J, Sorensen GL, Nikolic M, Frandsen R, Sorensen HB, Jennum P. Rapid eye movement sleep behavior disorder as an outlier detection problem. J Clin Neurophysiol 2014a;31:86-93.

Kempfner J, Sorensen HB, Nikolic M, Jennum P. Early automatic detection of Parkinson's disease based on sleep recordings. J Clin Neurophysiol 2014b;31:409-15.

Knudsen S, Gammeltoft S, Jennum PJ. Rapid eye movement sleep behaviour disorder in patients with narcolepsy is associated with hypocretin-1 deficiency. Brain 2010;133:568-79.

Koch H, Christensen JAE, Frandsen R, Zoetmulder M, Arvastson L, Christensen SR, et al. Automatic sleep classification using a data-driven topic model reveals latent sleep states. J Neurosci Methods 2014:235:130-7.

Latreille V, Carrier J, Lafortune M, Postuma RB, Bertrand JA, Panisset M, et al. Sleep spindles in Parkinson's disease may predict the development of dementia. Neurobiol Aging 2014. http://dx.doi.org/10.1016/j.neurobiolaging.2014.09.009. in press.

Latreille V, Carrier J, Montplaisir J, Lafortune M, Gagnon JF. Non-rapid eye movement sleep characteristics in idiopathic REM sleep behavior disorder. J Neurol Sci 2011;310:159-62.

Lu J, Sherman D, Devor M, Saper CB. A putative flip-flop switch for control of REM sleep. Nature 2006;441:589-94.

Luppi PH, Clément O, Sapin E, Gervasoni D, Peyron C, Léger L, et al. The neuronal network responsible for paradoxical sleep and its dysfunctions causing narcolepsy and rapid eye movement (REM) behavior disorder. Sleep Med Rev 2011;15:153-63.

Luppi PH, Clément O, Valencia Garcia S, Brischoux F, Fort P. New aspects in the pathophysiology of rapid eye movement sleep behavior disorder: the potential role of glutamate, gamma-aminobutyric acid, and glycine. Sleep Med 2013;14:714-8.
Micallef J, Rey M, Eusebio A, Audebert C, Rouby F, Jouve E, et al. Antiparkinsonian drug-induced sleepiness: a double-blind placebo-controlled study of L-dopa, bromocriptine and pramipexole in healthy subjects. Br J Clin Pharmacol 2009;67:333-40.

Munhoz RP, Teive HA. REM sleep behaviour disorder: How useful is it for the differential diagnosis of parkinsonism? Clin Neurol Neurosurg 2014;127:71-4.

Norman RG, Pal I, Stewart C, Walsleben JA, Rapoport DM. Interobserver agreement among sleep scorers from different centers in a large dataset. Sleep 2000;23:901-8.

Petit D, Gagnon JF, Fantini ML, Ferini-Strambi L, Montplaisir J. Sleep and quantitative EEG in neurodegenerative disorders. J Psychosom Res 2004;56:487-96.

Postuma RB, Gagnon JF, Rompré S, Montplaisir JY. Severity of REM atonia loss in idiopathic REM sleep behavior disorder predicts Parkinson disease. Neurology 2010;74:239-44.

Riemann D, Spiegelhalder K, Nissen C, Hirscher V, Baglioni C, Feige B. REM sleep instability - a new pathway for insomnia? Pharmacopsychiatry 2012;45: $167-76$.

Rodrigues Brazète J, Montplaisir J, Petit D, Postuma RB, Bertrand JA, Génier Marchand D, et al. Electroencephalogram slowing in rapid eye movement sleep behavior disorder is associated with mild cognitive impairment. Sleep Med 2013;14:1059-63.

Sakakibara R, Tateno F, Kishi M, Tsuyusaki Y, Terada H, Inaoka T. MIBG myocardial scintigraphy in pre-motor Parkinson's disease: a review. Parkinsonism Relat Disord 2014;20:267-73.

Salawu F, Danburam A, Olokoba AB. Non-motor symptoms of Parkinson's disease: diagnosis and management. Niger J Med 2010;19:126-31.

Saper CB, Chou TC, Scammell TE. The sleep switch: hypothalamic control of sleep and wakefulness. Trends Neurosci 2001;24:726-31.

Saper CB, Fuller PM, Pedersen NP, Lu J, Scammell TE. Sleep State Switching. Neuron 2010;68:1023-42.

Schapira AH, McDermott MP, Barone P, Comella CL, Albrecht S, Hsu HH, et al. Pramipexole in patients with early Parkinson's disease (PROUD): a randomised delayed-start trial. Lancet Neurol 2013;12:747-55.

Schenck CH, Bundlie SR, Mahowald MW. Delayed emergence of a parkinsonian disorder in $38 \%$ of 29 older men initially diagnosed with idiopathic rapid eye movement sleep behaviour disorder. Neurology 1996;46:388-93.

Schenck CH, Boeve BF, Mahowald MW. Delayed emergence of a parkinsonian disorder or dementia in $81 \%$ of older men initially diagnosed with idiopathic rapid eye movement sleep behavior disorder: a 16-year update on a previously reported series. Sleep Med 2013;14:744-8.

Schenck CH, Bundlie SR, Mahowald MW. REM behavior disorder (RBD): delayed emergence of parkinsonism and/or dementia in 65\% of older men initially diagnosed with idiopathic RBD, and an analysis of the minimum and maximum tonic and/or phasic electromyographic abnormalities found during REM sleep. Sleep 2003;26:A316.

Schwartz JR, Roth T. Neurophysiology of Sleep and Wakefulness: basic science and clinical implications. Curr Neuropharmacol 2008:6:367-78.

Sixel-Döring F, Trautmann E, Mollenhauer B, Trenkwalder C. Associated factors for REM sleep behavior disorder in Parkinson disease. Neurology 2011;77: 1048-54.

Sorensen GL, Mehlsen J, Jennum P. Reduced sympathetic activity in idiopathic rapid-eye-movement sleep behavior disorder and Parkinson's disease. Auton Neurosci 2013a;179:138-41.

Sorensen GL, Knudsen S, Jennum P. Sleep transitions in hypocretin-deficient narcolepsy. Sleep 2013b;36:1173-7.

Sorensen GL, Kempfner J, Zoetmulder M, Sorensen HB, Jennum P. Attenuated heart rate response in REM sleep behavior disorder and Parkinson's disease. Mov Disord 2012;27:888-94.

Stiasny-Kolster K, Mayer G, Schäfer S, Möller JC, Heinzel-Gutenbrunner M, Oertel WH. The REM sleep behavior disorder screening questionnaire-a new diagnostic instrument. Mov Disord 2007;22:2386-93.

Wienecke M, Werth E, Poryazova R, Baumann-Vogel H, Bassetti CL, Weller M, et al. Progressive dopamine and hypocretin deficiencies in Parkinson's disease: is there an impact on sleep and wakefulness? J Sleep Res 2012;21:710-7.

Zoetmulder M, Biernat HB, Nikolic M, Korbo L, Jennum PJ. Sensorimotor gating deficits in multiple system atrophy: comparison with Parkinson's disease and idiopathic REM sleep behavior disorder. Parkinsonism Relat Disord 2014a;20:297-302.

Zoetmulder M, Biernat HB, Nikolic M, Korbo L, Friberg L, Jennum PJ. Prepulse inhibition is associated with attention, processing speed, and 123I-FP-CIT SPECT in Parkinson's disease. J Parkinsons Dis 2014b;4:77-87. 